\title{
DESAFÍOS DE LA DESCONCENTRACIÓN Y PARTICIPACIÓN LOCAL EN EL MARCO DE LA PROTECCIÓN DE LOS DERECHOS EN SALUD: EXPERIENCIA DE LA INTENDENCIA MACRORREGIONAL NORTE, SUSALUD
}

\author{
Henry Rebaza-Iparraguirre ${ }^{1, a}$, Miguel Vela-López ${ }^{1, b}$, José Villegas-Ortega ${ }^{2,4, c}$, Michelle Lozada-Urbano²,d, \\ Oscar Munares-García $2,3, e$
}

\begin{abstract}
RESUMEN
En el Perú, la protección de los derechos en salud y la Superintendencia Nacional de Salud (SUSALUD) coexisten con la finalidad de que cada peruano ejerza sus derechos. Según el D.L. 1158, velar por el cumplimiento de los derechos de los usuarios requiere desplegar estrategias de desconcentración efectivas, así se crea la Intendencia Macro Regional Norte (IMRN), con sede en la ciudad de Chiclayo, que abarca Lambayeque, Tumbes, Piura, Cajamarca, La Libertad y Amazonas. En la macrorregión norte se realizan actividades de información, coordinación y participación local en salud, con énfasis en la atención de quejas y reclamos, así como la implementación y apoyo en el funcionamiento de juntas de usuarios (JUS). Luego de seis meses, la experiencia de la implementación de la IMRN se encuentra en proceso de consolidación.
\end{abstract}

Palabras clave: Regulación y fiscalización en salud; Fiscalización sanitaria, Derechos del paciente (fuente: DeCS BIREME).

\section{CHALLENGES TO DECENTRALIZATION AND LOCAL PARTICIPATION WITHIN THE HEALTH RIGHTS PROTECTION FRAMEWORK: EXPERIENCE OF THE SUSALUD NORTHERN MACRO-REGIONAL INTENDANCE}

\begin{abstract}
In Peru, health protection rights and the National Health Authority (SUSALUD) coexist with the aim to ensure that every Peruvian may exercise his/her rights. According to the L.D. (Spanish: Decreto legislativo) $N^{\circ} 1158$, strategies must be deployed to ensure effective decentralization and ensure compliance with user rights. As a result, the Northern Macro-regional Intendance (IMRN) was created, with headquarters in the city of Chiclayo, and a coverage area including Lambayeque, Tumbes, Piura, Cajamarca, La Libertad, and Amazonas. The northern macro-region promotes information dissemination, coordination, and local participation in health activities, with an emphasis on handling complaints and claims as well as implementation and operation support of user boards (JUS). After 6 months, implementation of the IMRN experience is undergoing consolidation.
\end{abstract}

Key words: Health care coordination and monitoring; Sanitary supervision; Patient rights (source: MeSH NLM).

\section{INTRODUCCIÓN}

Según el Ministerio de Salud, el derecho a la salud es considerado un derecho inclusivo que abarca la atención de la salud y sus determinantes ${ }^{(1)}$. Según la Organización Mundial de la Salud (OMS), el derecho a la salud incluye el acceso oportuno, aceptable y asequible a servicios de calidad suficiente ${ }^{(2)}$, y es la cobertura universal un medio para ejercer el derecho a la salud (3). En el reglamento de la Ley 29414, aprobado por el D.S. 027-2015-SA, se establecen los derechos de los usuarios de los servicios de salud y se designa a la Superintendencia Nacional de

\footnotetext{
Intendencia Macro Regional Norte, Superintendencia Nacional de Salud. Lambayeque, Perú.

Intendencia de Investigación y Desarrollo, Superintendencia Nacional de Salud. Lima, Perú

Facultad de Medicina Humana, Universidad Nacional Mayor de San Marcos. Lima, Perú

Facultad de Ingeniería de Sistemas, Universidad Nacional Mayor de San Marcos. Lima, Perú

Médico cirujano, magíster en Salud Pública; ${ }^{\mathrm{b}}$ médico cirujano; ${ }^{\mathrm{c}}$ licenciado en Computación, magíster en Ingeniería de Sistemas; ${ }^{\mathrm{d}}$ nutricionista, magíster en Economía de la Salud y las Políticas Sociales; ${ }^{\mathrm{e}}$ obstetra, doctor en Ciencias de la Salud

Recibido: 13/05/2016 Aprobado: 15/06/2016
}

Citar como: Rebaza-Iparraguirre H, Vela-López M, Villegas-Ortega J, Lozada-Urbano M, Munares-García O. Desafíos de la desconcentración y participación local en el marco de la protección de los derechos en salud: experiencia de la Intendencia Macrorregional Norte, SUSALUD. Rev Peru Med Exp Salud Publica. 2016;33(3):556-60. doi:10.17843/rpmesp.2016.333.2319 
Salud (SUSALUD) como entidad encargada de velar por la aplicación del reglamento mencionado. Los artículos 29 al 30 mencionan el derecho a presentar reclamos, la solución de controversias y la responsabilidad frente a la vulneración de derechos, respectivamente ${ }^{(4,5)}$.

Se denomina desconcentración al traslado de competencias técnicas y administrativas de un nivel jerárquico superior hacia un nivel inferior, para la toma de decisiones, sin que se pierda la relación organizacional y el nexo entre ambos (6,7). La desconcentración geográfica se da por dispersión del ámbito de acción de las instituciones públicas, e implica el traslado de las competencias a una unidad que por su ubicación puede ejercer mejor la función en un territorio ${ }^{(8)}$. SUSALUD planifica tener cinco intendencias macrorregionales (norte, centro, sur, oriente y Lima), ello ha sido plasmado en su reglamento (D.S. 008-2014-SA artículo 51), las cuales estarán ubicadas -además de Chiclayo-, en Loreto, Arequipa, Junín y Lima. La Intendencia Macrorregional Norte (IMRN) comprende seis regiones: Lambayeque, Tumbes, Piura, Cajamarca, La Libertad y Amazonas.

Dentro de las actividades estratégicas de SUSALUD, una primera etapa fue la implementación de tres intendencias macrorregionales: norte, sur y oriente, de cinco previstas, efectuado por Resolución de Superintendencia 111-2014-SUSALUD. La implementación de la IMRN no es una acción nueva, ya en Costa Rica (11), así como en Cuba ${ }^{(12)}$ se han desconcentrado algunas acciones similares a la de SUSALUD, pero no de la forma realizada en Perú. El objetivo del presente artículo es describir las acciones desconcentradas de la IMRN.

\section{ACCIONES DESCONCENTRADAS DE}

\section{LA IMRN}

La IMRN, se encuentra ubicada en la ciudad de Chiclayo, región Lambayeque, equidistante de las regiones de Tumbes, Cajamarca, Piura, La Libertad y Amazonas, según el reporte de marzo de 2016 cuenta con 12 trabajadores: asistente de gestión, técnico informático, especialistas en administración, protección de la salud, participación, atención rápida y legal, un asesor, un jefe zonal y un intendente.

Para alcanzar el adecuado proceso de desconcentración geográfica-funcional se debe pasar un proceso de fortalecimiento y consolidación, es así que en junio de 2014 se reglamentaron las funciones de SUSALUD; para el 2015 se inició el proceso de desconcentración en el norte del país, la decisión de iniciar en la zona norte se debió a la mayor cantidad de población, después de Lima, y a la disponibilidad de recursos. Se solicitó al Ministerio de Salud el presupuesto para la implementación de todas las intendencias macrorregionales, ello no fue posible, por lo que se reestructuró el presupuesto de SUSALUD, con la finalidad de iniciar en la zona norte. Para su inauguración se contó con la participación de las autoridades del gobierno regional de Lambayeque, así como autoridades locales y regionales. En el proceso de desconcentración funcional se asumen las actividades de vigilancia y prevención a través de la captación de quejas y reclamos y conformación de la Junta de Usuarios en Salud (JUS).

Una estrategia que el proceso incorpora son las acciones de participación local en salud, definida como un esfuerzo del poder de los ciudadanos, dirigido al desarrollo de comportamientos y ambientes saludables, e influye en las decisiones del sistema, puede ser a nivel macro: políticas sanitarias, priorización y planificación; a nivel meso: organización de servicios $y$, a nivel micro: provisión de intervenciones a usuarios y poblaciones ${ }^{(9)}$.

La participación local puede ser directa o indirecta. En la participación directa, el ciudadano, u otra persona, manifiesta su satisfacción con el sistema sanitario, captado a través de encuestas de satisfacción; así mismo, la recolección de quejas y reclamos, que sirven para detectar errores del sistema. La participación indirecta es la que se realiza a través de agentes $\mathrm{u}$ organismos que ostentan su representatividad (10), con el objetivo de que sus decisiones sean socialmente aceptadas.

\section{PARTICIPACIÓN DIRECTA}

El mecanismo de participación directa incluye actividades de consulta a través de encuestas, que son realizadas por la sede central de SUSALUD; la que actualmente se desarrolla es la denominada Encuesta Nacional de Satisfacción de Usuarios en Salud (ENSUSALUD) y cuya ejecución ha sido encargada al Instituto Nacional de Estadística e Informática (INEI), que debido a su representatividad nacional y regional permite contar con una radiografía de lo que está sucediendo en el sector salud en el país, por ser una actividad no desconcentrada, no se presenta información en este artículo. En cuanto a las quejas y reclamos, estas son canalizadas por varios medios: telefónico, correo electrónico, página web y redes sociales (Facebook y Twitter); también se cuenta con delegados, los cuales son profesionales de SUSALUD con presencia física en los principales hospitales públicos, los cuales recaban las consultas y quejas de los usuarios del sistema de salud público.

En el periodo de septiembre de 2015 a marzo de 2016, las quejas recolectadas fueron generadas por el Seguro Social de Salud (EsSalud), seguidos de los gobiernos regionales y Ministerio de Salud (Figura 1). La causa de queja más frecuente fue la falta de citas, demora o inadecuada atención en la consulta externa $(25,9 \%)$, le siguieron la 


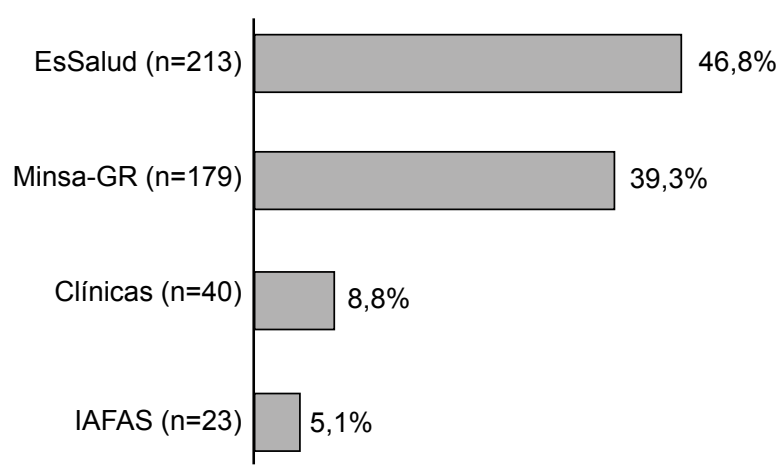

GR: gobiernos regionales, IAFAS: instituciones aseguradoras de fondos de aseguramiento en salud

Figura 1. Quejas atendidas en la Intendencia Macro Regional Norte, septiembre de 2015 a marzo de 2016, según institución donde se originó.

demora o disconformidad en el tratamiento quirúrgico $(10,3 \%)$ y demora o falta de necesidad de apoyo para trámites administrativos $(9,0 \%)$, estas tres causas representan el $45,2 \%$ de las quejas presentadas (Tabla 1 ).

Las quejas forman parte de los indicadores de calidad de atención, en la IMRN se ha podido determinar que son más de índole administrativo y quirúrgico, Valdés-Salgado et al. ${ }^{(13)}$ en México, identificó estos mismos problemas como causantes de quejas, Peña-Viveros et al. (14) identificó en hospitales de México, complicaciones durante la atención, error en el diagnóstico, utilización de estudios de gabinete, información deficiente, falta de vigilancia durante la hospitalización y revisiones vaginales múltiples en el trabajo de parto, como factores que se asociaron a las quejas.

Entre septiembre de 2015 a marzo de 2016 se han presentado 455 quejas, según el servicio de atención, siendo las relacionadas con la consulta externa (214) $\mathrm{y}$ las derivadas de problemas administrativos del sistema (113) las más numerosas, sumaron ambas 327 , de las 455 quejas. La mayor cantidad de quejas son presentadas en la región Lambayeque (365) y La Libertad (53), ello podría deberse a que la sede regional se encuentra en la región Lambayeque, teniendo mayor posibilidad de recabar quejas por proximidad. Según informes proyectados por el Instituto Nacional de Estadística e Informática (10), Lambayeque cuenta con población que supera el millón de habitantes, inferior a las regiones de La Libertad, Piura y Cajamarca, todas con porcentajes de filiación superiores al $80 \%$, excepto La Libertad que presentó $75,7 \%$, por lo que se esperaría cantidades similares de quejas, ello podría reflejar una recolección más eficiente en la región Lambayeque que en otras regiones. Finalmente, se indica que en promedio el porcentaje de solución de las quejas presentadas es de $86,6 \%$, siendo menor en Amazonas (Tabla 2).

\section{PARTICIPACIÓN INDIRECTA}

El mecanismo de participación indirecta se expresa por las JUS, la de Lambayeque está conformada por 24 miembros, $42 \%$ pertenecientes a grupos organizados (asociación de pacientes, adultos mayores y jóvenes) y $58 \%$ a miembros no agrupados (asegurados de EsSalud, SIS, discapacitados, usuarios de fuerzas armadas, policiales, comunidades locales, seguros privados y no asegurados), la actividad de los participantes fue fortalecida con reuniones de inducción. Así mismo, se conformó la Red de Apoyo Regional a la Junta de Usuarios (REDAJUS) Lambayeque, conformada por 16 representantes de instituciones y organizaciones de la sociedad civil, comprometidas con la promoción y protección de los derechos en salud, las coordinaciones entre la JUS y REDAJUS han permitido la elaboración del plan de trabajo de ambas. En Latinoamérica, Costa Rica, desde 1998, se implementó la JUS de manera desconcentrada, las cuales influyeron en la provisión y compras para su sistema de salud, inclinando la balanza en un sistema social de salud, con resultados favorables (11). Se ha determinado que cuando se empodera a la población, mediante los JUS, ello puede sostener y mejorar el acceso universal a los servicios de salud, como se ha demostrado en Costa Rica ${ }^{(15)}$.

Tabla 1. Quejas recibidas según causa en la Intendencia Macro Regional Norte septiembre de 2015 a marzo de 2016

\begin{tabular}{|c|c|c|}
\hline Causas & $\mathbf{n}$ & $\%$ \\
\hline $\begin{array}{l}\text { Falta de citas, demora o inadecuada } \\
\text { atención en consulta externa }\end{array}$ & 118 & 25,9 \\
\hline $\begin{array}{l}\text { Demora o disconformidad en tratamiento } \\
\text { quirúrgico }\end{array}$ & 47 & 10,3 \\
\hline $\begin{array}{l}\text { Demora o falta de necesidad de apoyo para } \\
\text { trámites administrativos }\end{array}$ & 41 & 9,0 \\
\hline $\begin{array}{l}\text { Problemas con actualización o desafiliación } \\
\text { al SIS/EsSalud }\end{array}$ & 37 & 8,1 \\
\hline $\begin{array}{l}\text { Demora o disconformidad en la atención de } \\
\text { hospitalización }\end{array}$ & 35 & 7,7 \\
\hline $\begin{array}{l}\text { Demora o disconformidad con exámenes } \\
\text { de apoyo diagnóstico }\end{array}$ & 34 & 7,5 \\
\hline Desabastecimiento de medicamentos & 30 & 6,6 \\
\hline $\begin{array}{l}\text { Insuficiente información sobre atención, } \\
\text { prestación o enfermedad }\end{array}$ & 29 & 6,4 \\
\hline Inadecuada atención & 26 & 5,7 \\
\hline Demora en la referencia & 24 & 5,3 \\
\hline Maltrato o falta de respeto a su dignidad & 14 & 3,1 \\
\hline $\begin{array}{l}\text { Demora o falta de atención en la } \\
\text { emergencia }\end{array}$ & 13 & 2,9 \\
\hline Equipamiento o infraestructura inadecuada & 3 & 0,7 \\
\hline Demora o disconformidad con reembolso & 2 & 0,4 \\
\hline Disconformidad en cobertura por la IAFA & 2 & 0,4 \\
\hline Total & 455 & 100,0 \\
\hline
\end{tabular}


Tabla 2. Quejas en instituciones prestadoras de servicios de Salud (IPRESS) e Institución Administradora de Fondos de Aseguramiento en Salud (IAFAS) y procedimientos realizados con gobiernos regionales (GR) por la Intendencia Macro Regional Norte, Superintendencia Nacional de Salud, septiembre 2015 a marzo de 2016

\begin{tabular}{|c|c|c|c|c|c|c|c|c|c|c|c|c|}
\hline \multirow{2}{*}{ Región } & \multicolumn{3}{|c|}{ Población * } & \multicolumn{7}{|c|}{ Procedencia de quejas y reclamos } & \multicolumn{2}{|c|}{ Procedimientos en GR } \\
\hline & Proyectada & Afiliada & $\%$ & CE & Hosp & Emerg & Admin & IAFAS & Total & \%Sol & Coordinación & Acciones \\
\hline Lambayeque & 1260650 & 1021953 & 81,1 & 180 & 62 & 29 & 82 & 12 & 365 & 89,0 & 1095 & 1825 \\
\hline La Libertad & 1859640 & 1407513 & 75,7 & 21 & 13 & 2 & 17 & - & 53 & 85,0 & 159 & 265 \\
\hline Piura & 1844129 & 1516779 & 82,2 & 8 & 6 & - & 9 & - & 23 & 74,0 & 69 & 115 \\
\hline Cajamarca & 1529755 & 1344031 & 87,9 & 3 & 2 & - & 1 & - & 6 & 83,0 & 18 & 30 \\
\hline Tumbes & 237685 & 203029 & 85,4 & - & - & - & 1 & - & 1 & 100,0 & 3 & 5 \\
\hline Amazonas & 422629 & 401933 & 95,1 & 1 & - & - & 1 & - & 2 & 50,0 & 6 & 10 \\
\hline Lima † & 9838251 & 7929452 & 80,6 & 1 & 2 & - & 2 & - & 5 & 80,0 & 15 & 25 \\
\hline Total & 16992739 & 13824690 & 81,4 & 214 & 85 & 31 & 113 & 12 & 455 & 86,6 & 1365 & 2275 \\
\hline
\end{tabular}

CE: consultorios externos, Hosp: hospitalización, Emerg: emergencia, Admin: administrativo, IAFAS: instituciones aseguradoras de fondos de aseguramiento en Salud, \%Sol: porcentaje de solución de quejas, GR: gobiernos regionales

* Población proyectada según INEI para el 2015 y población afiliada según reporte de afiliados de la Superintendencia Nacional de Salud a marzo de 2016

† Casos presentados en Lima y derivados a la intendencia Macro Regional Norte

\section{COORDINACIONES Y ACCIONES DE DIFUSIÓN (PROMOCIÓN DE DERECHOS)}

Para consolidar la estrategia participativa en los gobiernos regionales de la macrorregión norte, entre septiembre de 2015 a marzo de 2016 se realizaron 1365 coordinaciones, realizadas por correo electrónico, telefónico y personalmente con directores, jefes y responsables de áreas o servicios, la mayor cantidad de coordinaciones fueron realizadas en la región Lambayeque (1095) y la menor en Tumbes (Tabla 2). Esta estrategia forma parte de las actividades que realiza la IMRN y que se aúnan a las actividades programadas y no programadas que ha estado realizando, que ha permitido mantener la presencia de la Intendencia en las regiones, aunque con limitaciones de personal.

Asimismo, se han realizado 2275 acciones de difusión, que incluyen contenidos dirigidos a medios escritos, radiales, televisivos y a través de Internet (YouTube), como resultado, el público nos sigue, manifestando sus temas de interés, los cuales se retroalimentan en talleres de promoción y protección de los derechos en salud, prevención, restitución del derecho e investigación y desarrollo; dirigido a ciudadanos e instituciones regionales, como directores, gerentes, jefe de servicio médico, profesionales de la salud, de instituciones aseguradoras de fondos de aseguramiento en salud (IAFAS) e IPRESS públicas, privadas y mixtas, colegios profesionales de la salud, y profesionales en comunicación; ello nos ha permitido acercarnos a los grupos organizados de la región con la finalidad de relevar y poner en el centro al usuario.

\section{PERSPECTIVAS}

La experiencia de implementación de la IMRN después de seis meses, se encuentra en proceso de consolidación de la protección de los derechos de los usuarios, implementado a través de la participación directa e indirecta y su labor de información y coordinación, que ya muestra resultados, pero que las mismas están aún en marcha.

Las transformaciones regionales no deben ser una creación teórica por lo que resulta fundamental el aporte de todos quienes de una forma $u$ otra intervienen en el espacio territorial local. La suma de diferentes opiniones y la amplia participación serán un factor de éxito y respaldo a los cambios que deban implementarse, consideramos que este camino es posible porque se cuenta con normas técnicas a favor de la población y de intervención en las IPRESS y IAFAS públicas y privadas, así como nuestros equipos van ganando madurez operativa y la población irá empoderándose de sus derechos en salud.

El análisis estadístico de las quejas atendidas nos marca el camino a seguir con la desconcentración. En esta primera etapa es necesario alcanzar la integración entre equipos zonales y los servicios públicos y privados, que permitan implementar un modelo conjunto entre IPRESS públicas y privadas a partir de las necesidades de los pacientes, para unificar la promoción de derechos, necesarios para la gestión de quejas e intervenciones de buenos oficios. Consideramos que es necesario una mejor capacidad operativa y mejor asignación de recursos a nivel de capitales de región y en sus principales hospitales públicos. 
Las intendencias macrorregionales cuentan con jefaturas zonales a las que es necesario dotar de infraestructura adecuada que permita comunicación personalizada y virtual, para mejorar la conexión con los gobiernos regionales. Se han estimado que se requeriría alrededor de 28 jefaturas para su adecuado desarrollo. Así mismo, fortalecer la presencia de delegados en los principales hospitales, así como las JUS, REDAJUS y la acción en los centros de conciliación en salud.

Las coordinaciones entre la JUS y REDAJUS han permitido elaborar planes de trabajo de ambos. Este mecanismo de participación necesitarían ser mejor evaluado y faltaría la definición de los canales, para que esta JUS tenga influencia en las decisiones de salud de la región, que sería su elemento de participación plena. Se debe seguir trabajando en el posicionamiento de SUSALUD en los gobiernos regionales, sobre todo en la labor de promoción de derechos en salud, el funcionamiento de la intendencia y la protección de derechos en salud a nivel de regiones, debido a que estos últimos no han podido comprender al sistema de salud y su rol en ella, influyendo en la solución de problemas en las prestaciones de salud.

A manera de conclusión, indicamos que las acciones desconcentradas de la INMR ya a seis meses de su implementación, se encuentra en consolidación de las funciones de SUSALUD, con resultados satisfactorios y con perspectivas a futuro.

Contribuciones de autoría: HRI y MVL participaron en la redacción de la primera versión, JVO, OMG y MLU participaron en la concepción y diseño del artículo, JVO, OMG, MLU realizaron la redacción del manuscrito, la revisión crítica y aprobaron la versión final.

Fuente de financiamiento: autofinanciado

Conflictos de interés: no existen conflictos de interés

\section{REFERENCIAS BIBLIOGRÁFICAS}

1. Perú, Ministerio de Salud. Modelo de abordaje para la promoción de la salud [Internet]. Lima: MINSA; 2007 [Citado el 10 de mayo de 2016]. Disponible en: http://bvs.minsa.gob.pe/local/ promocion/203_prom30.pdf.

2. Organización Mundial de la Salud. Salud y derechos humanos. Centro de prensa. Nota descriptiva $\mathrm{N}^{\circ} 323$ [Internet]. Ginebra: OMS; 2015. [Citado el 8 de mayo de 2016]. Disponible en: http:// www.who.int/mediacentre/factsheets/ fs323/es/

3. Knaul FM, González-Pier E, GómezDantés O, García-Junco, D, ArreolaOrnelas $\mathrm{H}$, Barraza-Lloréns $\mathrm{M}$, et al. Hacia la cobertura universal en salud: protección social para todos en México. Salud Publica Mex. 2013;55(2):207-35.

4. Perú, Ministerio de Salud. Informe de Gestión - Los avances del proceso de implementación del Aseguramiento Universal en Salud (2009-2011). Lima: MINSA; 2011.

5. Perú, Ministerio de Salud. Decreto Legislativo que dispone medidas destinadas al fortalecimiento y cambio de denominación de la Superintendencia Nacional de Aseguramiento en Salud. Lima: MINSA; 2013.

6. García R. El proceso de desconcentración en el Ministerio de Salud de Costa
Rica: el caso de la Sub-Región Central Norte [tesis máster]. San José: Instituto Centroamericano de Administración Pública; 1990

7. Navarro R. Desconcentración administrativa y personalidad jurídica instrumental. San José: Gerencia del Proyecto de Modernización, CCSS; 2001.

8. Bolaños R. Conceptos transversales de organización gubernativa en Costa Rica: su aporte al desarrollo. Espacios Públicos. 2011;14(32):31-56.

9. Pineda CL. Los nuevos mecanismos de participación ciudadana en políticas de salud y su contribución a la igualdad y laequidad. In: XVII Congreso Internacional de CLAD sobre la reforma del Estado y de la Administración Pública. Cartagena, Colombia; 2012.

10. Sancho Serena F, Grané Alsina M, Olivet M. Sancho F, Grané M, Olivet M. Las estrategias de participación y representatividad local y ciudadana en la administración sanitaria. Med Clin (Barc). 2015;145(Supl 1):31-33. doi: 10.1016/S0025-7753(15)30035-X.

11. Sáenz M, Acosta M, Muiser J, Bermúdez J. Sistema de salud de Costa Rica. Salud Publica Mex 2011;53(Supl 2): S156-S167.

12. Álvarez A, García A, Rodríguez A, Bonet M, de Vos P, Van der Stuyff P. La regionalización de los servicios de salud como una estrategia de reorganización sanitaria. Rev Cubana Higiene Epidemiol 2008 abril [Citado el 8 de mayo de 2016];46(1): [13 pág.] Disponible en: http://www.redalyc.org/articulo. oa? id=223218855007

13. Valdés-Salgado R, Molina-Leza J, SolísTorres C. Aprender de lo sucedido. Análisis de las quejas presentadas ante la Comisión Nacional de Arbitraje Médico. Salud Publica Mex 2001;43(5):444-54.

14. Peña-Viveros R, Rodríguez-Moctezuma J, López-Carmona J. Factores asociados a la gestión de quejas contra médicos que laboran en hospitales del Instituto Mexicano del Seguro Social. Salud Publica Mex. 2004;46(3):210-5.

15. Chávez B, Montoya Y. Comparación de las reformas de los sistemas de salud en Colombia y Costa Rica en su dimensión política. Rev Facult Nac Salud Publica. 2011;29(1):65-74.

Correspondencia: Oscar Fausto Munares García

Dirección: Av. Velasco Astete 1398, Santiago de Surco.

Teléfono: (+511) 3726150 anexo: 5714

Correo electrónico:omunaresg@unmsm.edu.pe 